\title{
Method of Mathematical Description for Digital System Blocks Logical Models
}

\author{
S.V. Gavrilov, A.D. Ivannikov, A.L. Stempkovsky \\ Institute for Design Problems in Microelectronics of Russian Academy of Sciences, ippm@ippm.ru
}

\begin{abstract}
Digital systems blocks are considered. We consider that the functioning of such blocks can be represented as a sequence of functions of a finite alphabet. Digital systems are simulated on the logic level of the signals they exchange with external environment. For a system design debugging by simulation it is necessary to generate the set of tests for applying to the simulated system to verify the correctness of functioning. The most productive and economical test set could be generated if a formal description of digital block or system possible input data domain is known. The input data domain structure are analyzed and described for digital blocks and systems with finite alphabet of functions. The formal description of input data domain for each function of digital block or system are proposed. Proposed description has the form of labeled directed graph describing the sequence of input logical signals with timing constrains.
\end{abstract}

Keywords - digital block formal models, input interaction domain, design debugging, computer-aided design.

\section{INTRODUCTION}

For digital system design development and debugging the method of computer simulation is widely used. Some input signal sequences are applied to the computer model of a digital system, and the reaction of the system model being designed is checked for compliance with the required one $[1,2]$

An important task here is to select a finite number of time-finite test input interactions (test cases) [3, 4]. With the increasing of complexity of the digital systems being designed and, correspondingly, the increasing of complexity and duration of testing of its projects, the task of selecting a minimal complete, in a certain sense, test set, verifying the absence of design errors, is becoming more and more urgent $[5,6]$.

For the test cases generation it is necessary to have the permissible input interaction set for a developing digital system as a whole and for its blocks as well $[6,7]$. The goal of this research is the development of digital system block models, first of all from the point of view of permissible input interaction descriptions.

\section{DESCRIPTION OF DIGITAL CONTROL SYSTEM MODEL USED}

The interaction of a digital system with the controlled equipment and the outside world in general is done through external lines and buses - the sets of lines through which homogeneous information is transmitted, for example, addresses, or data. And in digital systems, bi-directional buses and lines, which also have a state with a high output resistance (disconnected state), are widely used. We will consider the logical model of signals on the buses and lines of digital systems, that is, assume that the signal values are represented as 0 or 1 on the lines and as a number from 0 $2^{n}-1$ range on the system buses. Let us call digital signals of external buses and lines by the term terminal variables set $\mathbf{P}$. Variable $p \in \mathbf{P}$ always has one of the values of finite set $\mathbf{Z}_{p}$ whose elements define both the integer value of the signal and the directivity of the bus or line operation.

An event in variable $p$ is the change of its value from value $z_{1} \in \mathbf{Z}_{p}$ to value $z_{2} \in \mathbf{Z}_{p}$ at time $t$. We denote such an event $\chi_{p, z_{1}, z_{2}}^{t}$. The interaction of a digital system with the external environment, including the controlled object, is the sequence of signal switching on the terminal buses and lines, that is, the sequence of events. For each system being designed, there is a set of $\Psi$ admissible interactions with the external environment, each of which is a map $\psi$. $[0, t) \rightarrow \mathbf{Q}, t \in \mathbf{T}, \mathbf{Q}=\prod_{p \in \mathbf{P}} \mathbf{Z}_{p}$.

In digital systems, for each finite time interval, the number of events by terminal variables, that is, the number of changes in their values, is finite. Consequently, any interaction $\psi$ can be represented as a vector $\left(z_{p_{1}, \ldots,}^{i n} z_{p_{k}}^{i n}\right)$ of the initial values of variables $p_{1}, \ldots, p_{k}(k-$ cardinality of set $\mathbf{P}$ ) at time $t=0$ and the sequence of events with respect to the variables of set $\mathbf{P}$ with a finite number of events in any finite time interval.

If in the sequence of events only events, being changes in input signals values, are selected, then such a sequence can be called the input action. However, the timing of input signals to the digital system is often determined by the system's readiness to accept these signals, as indicated by certain output signals of the system. Performing of an operation, for example, reading data by a digital system can be initiated not by signals from the external environment, but by the system itself. Therefore, the use of input influences as arguments for the operation of a digital system is not always convenient.

From the sequence of events of interaction $\psi$, we select a sequence of input events and output exchange control events, which, according to the specified exchange protocol, cause the timing of the input events. We call this sequence the input interaction:

$$
\mu=\left(z_{p_{1}, \ldots,}^{\text {in }} z_{p_{n+q}}^{\text {in }}\right), \chi_{p_{i_{1}}, z_{j_{1}}, z_{j_{2}}}^{t_{1}}
$$




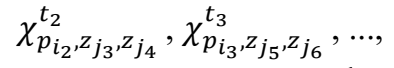

where $t_{1} \leq t_{2} \leq t_{3} \leq \ldots-$ ordered sequence of event times;

$p_{i_{1}}, p_{i_{2}}, p_{i_{3}}, \ldots-$ variables belonging to set $\mathbf{P}$;

$z_{j_{1}}, z_{j_{3}}, z_{j_{5}}, \ldots-$ values of variables just before the event;

$z_{j_{2}}, z_{j_{4}}, z_{j_{6}}, \ldots-$ values of variables immediately after the event;

$$
\chi_{p_{i_{1}}, z_{j_{1}}, z_{j_{2}}}^{t_{1}}, \chi_{p_{i_{2}}, z_{j_{3}}, z_{j_{4}}}^{t_{2}}, \chi_{p_{i_{3}}, z_{j_{5}}, z_{j_{6}}}^{t_{3}} \ldots-\text { - input events }
$$
and output events of exchange control;

$t_{1} \leq t_{2} \leq t_{3} \leq \ldots-$ ordered sequence of times of input interaction events.

In the model under consideration, input interactions are used as arguments to the functioning of digital systems that makes it possible to consider operating modes initiated by both external input signals and by the digital systems themselves. In more detail, a formal representation of the admissible interactions of digital systems is considered in $[8,9]$.

All above description is valid not only for digital systems as a whole, but for digital system blocks as well.

\section{THE STRUCTURE OF THE SET OF ADMISSIBLE INPUT INTERACTIONS}

Each digital system block during its operation performs some sequence of functions (operations) of a finite alphabet of functions $\mathbf{K}$, proper to a particular system block. The execution of each function is caused by one of input interactions of a particular class, and each input interaction of this class contains a finite number of events.

Let $f$ be a finite sequence of functions, and $\mathbf{F}$ generally countable set of finite sequences $f$. Each sequence of functions $f$ starting from the time $t=0$ (for example, power on) is specified, at least by one input interaction $\mu^{f} \in \mathbf{M}$. This follows from the fact that $\mathbf{M}$ contains all the valid input interactions for any permissible sequence of functions of the digital system block or digital system as a whole.

In most cases, the same functions can be performed by a digital system with different sets of data, and it causes determination of same sequence of functions $f$ by various $\mu$. Due to the fact that for the various samples of a digital system the delays of the output events of exchange control relative to input events vary within certain limits, as well as in connection with the permissibility of varying timings of input events with respect to each other and to the output events of exchange control, set $\mathbf{M}$ contains a continual subset $\mathbf{M}^{f} \subset \mathbf{M}$ of input interactions each of which causes the execution of the final sequence of functions $f$ by the digital system. A set of input interactions can be represented as:

$$
\mathbf{M}=\bigcup_{f \in \mathbf{F}} \mathbf{M}^{f} ; \mathbf{M}^{f^{\prime}} \cap \mathbf{M}^{f^{\prime \prime}}=\varnothing \text { if } f^{\prime} \neq f^{\prime \prime} .
$$

Input interaction $\mu \in \mathbf{M}^{f}$ contains a finite set of events

$$
\left\{\chi_{p_{i_{1}}, z_{j_{1}}, z_{j_{2}}}^{t_{1}}, \ldots, \chi_{p_{i_{n}}, z_{j_{2 n-1}}, z_{j_{2 n}}}^{t_{n}}\right\}
$$

$$
\text { where } \quad n \text {-number of events in } \mu \text {. }
$$

Set $\mathbf{M}^{f}$ also contains the input interactions, in which times of events differ within certain limits. The limitations of these differences can be defined as

$$
\begin{gathered}
t_{\text {min }}^{l, m} \leq t_{m}-t_{l} \leq t_{\text {max }}^{l, m},(l, m) \in \mathbf{C}, \\
\mathbf{C} \subset\{1,2, \ldots, n\} \times\{1,2, \ldots, n\},
\end{gathered}
$$

where $\quad t_{\min }^{l, m}, t_{\max }^{l, m}-$ minimum and maximum permissible intervals between $l$-th and the $m$-th events;

C - a finite set of pairs of events of set (1) for which the time limits are specified.

Input interaction $\mu$ of set $\mathbf{M}^{f}$, except the events (3), may contain (within certain time intervals) indifferent events that do not affect the digital system operation. Suitable for the practice method of specification of whole class $\mathbf{M}^{f}$ should be considered separately, but now we note that in the set of constraints (3) there are times of significant events, i.e., the events of set (1).

Let us distinguish in $\mathbf{C}$ all pairs $(l, m)$ for which $t_{m}$ is a time of output event of exchange control and present them as set $\mathbf{C}_{\text {out }}$, and all pairs $(l, m)$ for which $t_{m}$ is a time of input events as set $\mathbf{C}_{\text {in }}$. Then the restrictions on the moments of time of exchange output events are

$$
t_{\text {min }}^{l, m} \leq \mathrm{t}_{\mathrm{m}}-\mathrm{t}_{1} \leq t_{\text {max }}^{l, m},(l, m) \in \mathbf{C}_{\text {out }},
$$

and the restrictions on the moments of time of input events are:

$$
t_{\text {min }}^{l, m} \leq t_{m}-t_{l} \leq t_{\text {max }}^{l, m},(l, m) \in \mathbf{C}_{\text {in. }}
$$

Consider space $\mathbf{G}=\prod_{\text {cout }}\left\{t_{m}-t_{l} \mid t_{m}-t_{l} \geq 0\right\}$. Each point of $g \in \mathbf{G}$ defines the specific values of the delays of exchange control output events. In the space of $\mathbf{G}$ let us mark area $\mathbf{G}_{f} \in \mathbf{G}$, for all points of which the limitations (2) are valid. Area $\mathbf{G}_{f}$ defines the delays of exchange control output events, permitted under the technical project. If the technical task consistently (and we consider just the case), then $\mathbf{G}_{f} \neq \varnothing$.

For any point $g \in \mathbf{G}_{f}$ in connection with the permissibility of such delays of exchange control output events there exists a non-empty set of input interactions $\mathbf{M}_{g}^{f}$ ensuring the fulfillment of sequence of functions $f$ by the system or its block:

$$
\mathbf{M}^{f}=\bigcup_{g \in \mathbf{G}_{f}} \mathbf{M}_{g}^{f}, \mathbf{M}_{g}^{f} \neq \varnothing,
$$

where $\quad \mathbf{M}_{g}^{f}-\mathrm{a}$ set of input interactions, ensuring the fulfillment of a finite sequence of functions $f$ by the digital system while there exist fixed delays of exchange control output events defined by $g \in \mathbf{G}_{f}$.

Thus, the set of permissible input interactions can be represented as

$$
\mathbf{M}=\bigcup_{f \in \mathbf{F}} \mathbf{M}^{f} ; \mathbf{M}^{f^{\prime}} \cap \mathbf{M}^{f^{\prime \prime}}=\varnothing \text { when } f^{\prime} \neq f^{\prime \prime \prime} ;
$$




$$
\mathbf{M}^{f}=\cup_{g \in \mathbf{G}_{f}} \mathbf{M}_{g}^{f} ; \mathbf{G}_{f} \neq \varnothing ; \mathbf{M}_{g}^{f} \neq \varnothing \text { when } f \in \mathbf{F} .
$$

In such a way we have defined permissible input interaction set, e.g. the structure of possible arguments for digital block or system functioning.

\section{INPUT INTERACTION SET DEFINITION FOR EACH FUNCTION}

Let us consider the way of definition for input interaction set $\mathrm{M}^{k}$ causing the fulfillment of digital block function $k$.

Taking into account (1) and (2) each $\mu, \mu \in \mathrm{M}^{k}$ could be written as:

$$
\begin{gathered}
\left(\left(z_{p_{1}}^{\text {in }}, \ldots, z_{p_{m}}^{\text {in }}\right),\left\{\left(t_{1}, p_{i_{1}}, z_{1}^{\prime}, z_{1}^{\prime \prime}\right),\left(t_{2}, p_{i_{2}}, z_{2}^{\prime}, z_{2}^{\prime \prime}\right), \ldots,\right.\right. \\
\left.\left.\left(t_{n}, p_{i_{\mathrm{n}}}, z_{n}^{\prime}, z_{n}^{\prime \prime}\right)\right\}\right),
\end{gathered}
$$

where $z_{p_{1}}^{\text {in }}, \ldots, z_{p_{m}}^{\text {in }}-$ initial variable values;

$t_{i}, p_{i_{i}}, z_{i}^{\prime}, z_{i}^{\prime \prime}$ - the description for $i$-th event.

Taking this into account let us try to define the whole $\mathrm{M}^{k}$ set as:

$$
\left(\tilde{\mathbf{Z}}_{\mathbf{p}_{1}}^{\text {in }}, \ldots, \tilde{\mathbf{Z}}_{\mathbf{p}_{m}}^{\text {in }}\right),\left\{\left(\theta_{1}, p_{i_{1}}, \tilde{\mathbf{Z}}_{1}^{\prime}, \tilde{\mathbf{Z}}_{1}^{\prime \prime}\right),\left(\theta_{2}, p_{i_{2}}, \tilde{\mathbf{Z}}_{2}^{\prime}, \tilde{\mathbf{Z}}_{2}^{\prime \prime}\right), \ldots,\right.
$$
$\left.\left(\theta_{n}, p_{i_{n}}, \tilde{\mathbf{Z}}_{n}^{\prime}, \tilde{\mathbf{Z}}_{n}^{\prime \prime}\right)\right\}$,

$$
t_{\text {min }}^{l, q} \leq \theta_{q}-\theta_{l} \leq t_{\text {max }}^{l, q},(l, q) \in \mathbf{C},
$$

where $\theta_{1}, \theta_{2}, \ldots, \theta_{n}-$ event times;

$\tilde{\mathbf{Z}}_{\mathbf{p}_{i}}^{\text {in }}, \quad i=1,2, \ldots, m-$ possible initial value subset of variables of set $\mathbf{P}^{\prime} \cup \mathbf{P}^{0}$;

$m$ - cardinality of the set $\mathbf{P}^{\prime} \cup \mathbf{P}^{0}$;

$p_{i_{1}}, p_{i_{2}}, \ldots, p_{i_{n}}-$ belong to the set $\mathbf{P}^{\prime} \cup \mathbf{P}^{0}$;

$\tilde{\mathbf{Z}}_{1}^{\prime}, \tilde{\mathbf{Z}}_{2}^{\prime}, \ldots, \tilde{\mathbf{Z}}_{n}^{\prime}$ - possible value subset of variables $p_{i_{1}}, p_{i_{2}}, \ldots, p_{i_{n}}$ before the event;

$\tilde{\mathbf{Z}}_{1}^{\prime \prime}, \tilde{\mathbf{Z}}_{2}^{\prime \prime}, \ldots, \tilde{\mathbf{Z}}_{n}^{\prime \prime}$ - possible value subset of variables $p_{i_{1}}, p_{i_{2}}, \ldots, p_{i_{n}}$ after the event;

C - set of event pairs for which time restrictions are defined.

Input interaction $\mu$, defined as (5), belongs to $\mathrm{M}^{k}$, defined as (6), if:

a) $z_{p_{1}}^{\text {in }} \in \tilde{\mathbf{Z}}_{\mathbf{p}_{1}}^{\text {in }}, \ldots, z_{p_{m}}^{\text {in }} \in \tilde{\mathbf{Z}}_{\mathbf{p}_{m}}^{\text {in }}$

b) there exists an isomorphism for the set of events (5) into the set of quadruples in (6) such that the corresponding events have the same names of variables: $z_{1}^{\prime} \in \tilde{\mathbf{Z}}_{1}^{\prime}, \ldots z_{n}^{\prime} \in \tilde{\mathbf{Z}}_{n}^{\prime}, z_{1}{ }^{\prime \prime} \in \tilde{\mathbf{Z}}_{1}^{\prime \prime}, \ldots, z_{n}^{\prime \prime} \in \tilde{\mathbf{Z}}_{n}^{\prime \prime} \quad, \quad$ and restrictions of (6) are valid to event times $t_{1}, \ldots, t_{n}$.

Above description of $\mathrm{M}^{k}$ and subsets $\tilde{\mathbf{Z}}_{\mathbf{P}_{i}}^{\mathrm{H}}, \tilde{\mathbf{Z}}^{\prime}, \tilde{\mathbf{Z}}^{\prime \prime}$ introduction make it possible to define input interaction sets with different data sets.

Let us assume that terminal variable set $\mathbf{P}$ is defined, each variable has value set $\mathbf{Z}_{p}$. For each variable $p$ let us consider a value alphabet $\mathbf{3} p=\mathbf{Z}_{p} \cup$ $\left\{z_{i j} \mid\left(z_{i} \in \mathbf{Z}_{p}\right) \&\left(z_{j} \in \mathbf{Z}_{p}\right) \&\left(z_{i} \neq z_{j}\right)\right\}$, where $z_{i j}-$ transaction from $z_{i}$ to $z_{j}$. In the alphabet $\boldsymbol{3}_{p}$ we select non-empty subsets $\boldsymbol{3}_{l}^{p} \subset \mathbf{3}_{p}$ such that $\mathbf{3}_{p}=\bigcup_{l} \mathbf{3}_{l}^{p}$. For example if bus signal value could be $0-255$ or $\$(\$-$ high impedance state), it is possible to use the following subsets:

$$
\boldsymbol{3}_{l}: \boldsymbol{3}_{1}=\{\$\}, \quad \boldsymbol{3}_{2}=\{0,1, \ldots, 255\}, \quad \mathbf{3}_{3}=\boldsymbol{3}_{2} \cup
$$
$\left\{z_{i j} \mid\left(z_{i} \in \mathbf{3}_{2} \cup \mathbf{3}_{1}\right) \&\left(z_{j} \in \mathbf{3}_{2} \cup \mathbf{3}_{1}\right)\right\}, \quad \mathbf{3}_{4}=\mathbf{3}_{1} \cup \mathbf{3}_{2} \cup$ $\mathbf{3}_{3}$.

In the above equation $\mathbf{3}_{2}-$ stable data value, $\mathbf{3}_{4}-$ non-stable data value.

Subsets $\boldsymbol{3}_{l}^{p}, l=1,2, \ldots$ form finite alphabet $\widehat{\boldsymbol{Z}}_{p}$. Each set $\mathrm{M}^{k}$ could be expressed as finite event set in the alphabet $\widehat{\mathbf{3}}_{p}$. On the event set of the set $\mathrm{M}^{k}$ a partial order over time is defined. So it is naturally to represent $\mathrm{M}^{k}$ by oriented graph $\mathcal{G}^{k}\left(\mathbf{V}^{k}, \mathbf{E}^{k}\right)$, each its vertex $v \in \mathbf{V}^{k}$ corresponds to a variable value change from one $\mathbf{3}_{l}^{p}$ to another. Let us mark each vertex $v, v \in \mathbf{V}^{k}$ by variable $p$ and by sets $\mathbf{3}_{l}^{p}, \mathbf{3}_{l^{\prime}}^{p}$, if a vertex $v$ represents changing variable $p$ value from $\mathbf{3}_{l}^{p}$ to $\mathbf{3}_{l^{\prime}}^{p}$.

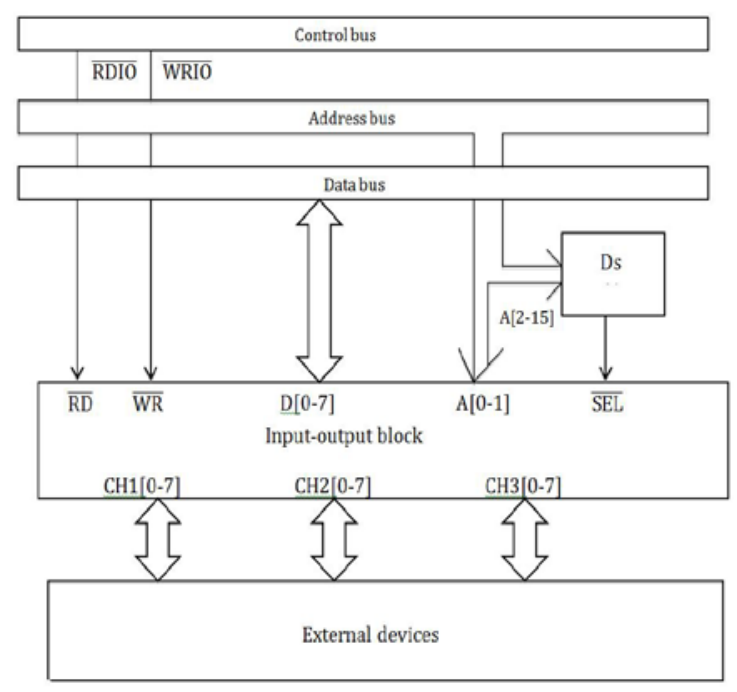

a)

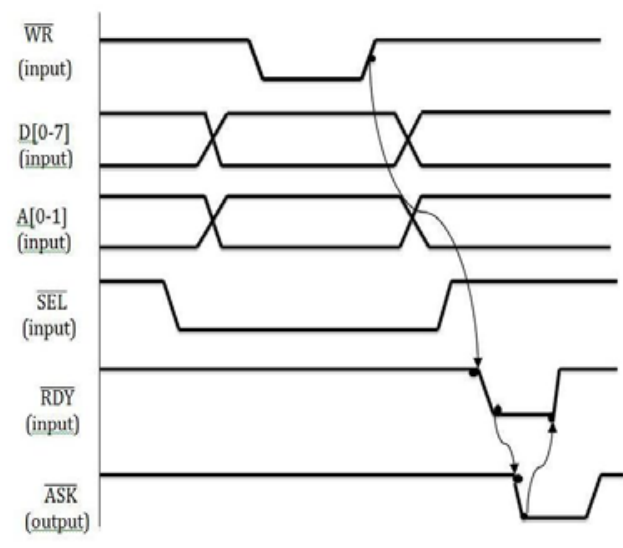

b)

Fig. 1. Parallel input-output digital block: a) connection, b) timing diagram 
As an example let us consider some digital parallel input-output interface block (Fig. 1) For block function control the following signals are used: $\mathrm{D}[0-7]$ - data bus; $\mathrm{A}[0-1], \mathrm{A}[2-15]$ - address bus; RES, $\overline{\mathrm{RD}}, \overline{\mathrm{WR}}$ - reset, reed and write signals; $\overline{\mathrm{SEL}}, \overline{\mathrm{RDY}}, \overline{\mathrm{ASK}}$ - select, ready, acknowledgement signals; $\mathrm{CH} 1, \mathrm{CH} 2, \mathrm{CH} 3$ - channel buses with corresponding bit numbers.

On the vertex set $\mathbf{V}_{p}^{k}$, describing variable $p$ value changing a partial order over time is defined. This order maps onto edge set $\mathbf{E}^{k}$. Each edge has two marks $t_{\min }, t_{\max }$, $0 \leq t_{\min } \leq t_{\max } \leq \infty$

Input interaction set $\mathrm{M}^{k}$ for data writing to the described digital block could be determined by the graph (Fig. 2). For this case:

$\mathbf{P}=\{\mathrm{A}, \mathrm{D}, \overline{\mathrm{SEL}}, \overline{\mathrm{WR}}, \overline{\mathrm{RDY}}, \overline{\mathrm{ASK}}\} ;$

$\mathbf{Z}_{\mathrm{A}}=\{0,1,2,3, \$\} ; \mathbf{Z}_{\mathrm{D}}=\{0,1, \ldots, 255, \$\} ; \mathbf{Z}_{\overline{\mathrm{RDY}}}=\{0,1\} ;$ $\mathbf{Z}_{\overline{\mathrm{WR}}}=\{0,1\} ; \mathbf{Z}_{\overline{\mathrm{RDY}}}=\{0,1\} ; \mathbf{Z}_{\overline{\mathrm{ASK}}}=\{0,1\}$;

$\widehat{\boldsymbol{Z}}_{\mathrm{A}}=\{\mathrm{ST}, \mathrm{NST}, \mathrm{HI}, \mathrm{X}\}, \mathrm{ST}=\{0,1,2,3\} ; \mathrm{NST}=\mathrm{ST} \cup$ $\left\{\chi_{i, k} \mid z_{i} \neq z_{k}, z_{i} \in \mathrm{ST}, z_{k} \in \mathrm{ST}\right\} ; \mathrm{HI}=\{\$\} ; \mathrm{X}=\mathrm{NST} \cup H \mathrm{I} \cup$ $\left\{\chi_{i, \$} \mid z_{i} \in \mathrm{ST}\right\} \cup\left\{\chi_{\$, i} \mid z_{i} \in \mathrm{ST}\right\} ;$

$\widehat{\mathbf{3}}_{D}=\{\mathrm{ST}, \mathrm{NST}, \mathrm{HI}, \mathrm{X}\}, \mathrm{ST}=\{0,1, \ldots, 255\} ;$

$\mathrm{NST}=\mathrm{ST} \cup\left\{\chi_{i, k} \mid z_{i} \neq z_{k}, z_{i} \in \mathrm{ST}, z_{k} \in \mathrm{ST}\right\} ;$

$\mathrm{HI}=\{\$\}, \mathrm{X}=\mathrm{NS} \cup H \mathrm{I} \cup\left\{\chi_{i, \$} \mid z_{i} \in \mathrm{ST}\right\} \cup\left\{\chi_{\$, i} \mid z_{i} \in \mathrm{ST}\right\} ;$

$\widehat{\mathbf{3}} \overline{\mathrm{SEL}}=\{0,1, X\}, X=\left\{0,1,3_{0,1}, 3_{1,0}\right\}$;

$\widehat{3} \overline{\mathrm{WR}}=\{0,1, X\}, X=\left\{0,1,3_{0,1}, 3_{1,0}\right\}$;

$\widehat{\mathbf{Z}}_{\overline{\mathrm{RDY}}}=\{0,1, \mathrm{X}\}, \mathrm{X}=\left\{0,1,3_{0,1}, 3_{1,0}\right\}$;

$\widehat{3} \overline{\mathrm{ASK}}=\{0,1, X\}, X=\left\{0,1,3_{0,1}, 3_{1,0}\right\}$.

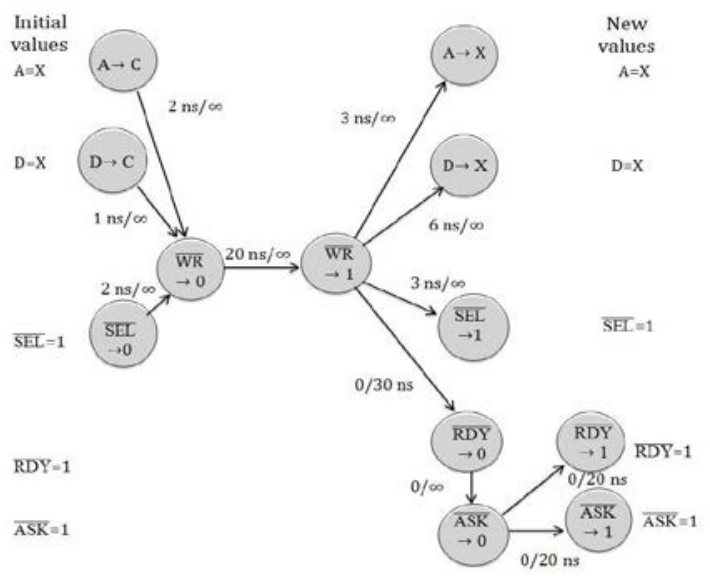

Fig. 2. Input interaction set definition for the function of data writing to parallel input-output block

Graph vertexes are denoted by variable names and their new values (Fig. 2). If variable initial values in the $\widehat{\mathbf{3}}_{p}$ alphabet are known the graph determines the input interaction set for a function. Having graph $\mathcal{G}^{k}\left(\mathbf{V}^{k}, \mathbf{E}^{k}\right)$ for each $\mu$ (1) it is possible to determine if $\mu$ belongs to the set $\mathrm{M}^{k}$ or not.

Graph $\mathcal{G}^{k}\left(\mathbf{V}^{k}, \mathbf{E}^{k}\right)$ describes the set $\mathrm{M}^{k}$ formally and is more understandable then timing diagram (Fig. 1b), which is usually used for an explanation of digital block functioning.

\section{CONCLUSION}

Graph $\mathcal{G}^{k}\left(\mathbf{V}^{k}, \mathbf{E}^{k}\right)$ defines input interaction set for a digital block function $k$. The described above algorithm enables us to define input interaction set for each function of a digital system as whole as well.

Such a graph together with (4) defines permissible input interaction set both for each separate digital block of a system and for a digital system as a whole. Permissible input interaction set structure is an input data for debugging test set generation. Those tests are used for digital system design debugging by computer simulation.

\section{ACKNOWLEDGMENTS}

With the support of Russian Foundation for Basic Research (Grant \# 17-07-00683)

\section{REFERENCES}

[1] Y. Lin, A. Su, "Functional Verifications for SoC Software/Hardware Co-Design: From Virtual Platform to Physical Platform," 2011 IEEE International SoC Conference (SOCC), pp. 201-206.

[2] S.V. Gavrilov, O.N. Gudkova, A.L. Stempkovskiy, "The Analysis of the Performance of Nanometer Intellectual Property Blocks Based on Interval Simulation," Russian Microelectronics, vol. 42, no. 7, 2013, pp. 396-402.

[3] S. Jain, P. Govani, K.B. Poddar, A.K. Lal, R.M. Parmar, "Functional Verification of DSP Based On-Boad VLSI Design," Internftional Conftrence on VLSI Systems, Architectures, Technology and Applications (LSI-SATA), 2016, pp. 1-4.

[4] A.M. Cruz, R.B. Fernandez, H.M. Lozano, M.A. Ramirez Salinas, L.A. Vila Vargas, "Automated Functional Test Generation for Digital Systems Through a Compact Binary Differential Evolution Algorithm," Journal of Electronic Testing-Theory and Applications, 2015, vol. 31, no. 4, pp. 361-380.

[5] A. Kamkin, "Simulation-Based Hardware Verification with Time-Abstract Models," Proceedings of IEEE East-West Design and Test Symposium (EWDTS'2011), pp. 43-47.

[6] A. Ivannikov, B. Pozdneev, I. Romanova, S. Tumkovskiy, "Debugging test set generation for digital control system functions", 2018 Moscow Workshop on Electronic and Networking Tehnologies (MWENT), 2018, pp. 1--5.

[7] J. Shi, W. Liu, M. Jiang, "Software Hardware CoSimulation and Co-Verification in Safety Critical System Design," 2013 IEEE International Conference on Intelligent Rail Transportation (ICIRT), pp. 71-74.

[8] A. Ivannikov, A. Romanov, A. Stempkovsky, "Set-theoretic Model of Digital Systems Functioning", International Siberian Conference on Control and Communications (SIBCON), IEEE, Moscow, 2016. Article Number: 308fu4t.

[9] A. Ivannikov, V. Kulagin, A. Romanov, B. Pozdneev, "Algebraic Models of Digital System Design Debugging Decomposition", Proceedings of 2016 IEEE East-West Design and Test Symposium (EWDTS 2016), pp. 1-4. 